\title{
Radiotherapy may improve survival of ES-SCLC with distant metastasis only for patients with one metastatic site: A population-based study
}

\author{
XIAOLING SHANG ${ }^{1,2^{*}}$, JIAMAO LIN $^{3 *},{\text { ZHENXIANG } \mathrm{LI}^{3} \text { and HAIYONG WANG }}^{3}$ \\ ${ }^{1}$ Department of Clinical Laboratory, Shandong University, Jinan, Shandong 250012; Departments of ${ }^{2}$ Clinical Laboratory \\ and ${ }^{3}$ Internal Medicine-Oncology, Shandong Cancer Hospital and Institute, Shandong First Medical University \\ and Shandong Academy of Medical Sciences, Jinan, Shandong 250117, P.R. China
}

Received February 18, 2019; Accepted September 6, 2019

DOI: $10.3892 / \mathrm{ol} .2019 .11092$

\begin{abstract}
The aim of the present study was to investigate the prognostic impact of RT on patients with extensive stage small cell lung cancer (ES-SCLC) and distant metastasis. Using the Surveillance Epidemiology and End Results (SEER) database, 8,595 patients with ES-SCLC exhibiting distant metastasis treated between 2010 and 2013 were identified. Patient baseline characteristics were compared using the $\chi^{2}$ test. The Kaplan-Meier test was used to analyze subgroup cancer-specific survival (CSS) rate, and differences were compared using a log-rank test. Univariate and multivariate Cox regression models were used to analyze the prognostic variables on CSS. RT was determined to be an independent prognostic factor for patient CSS $(\mathrm{P}<0.001)$. In addition, RT could improve the CSS of patients with ES-SCLC with one metastatic lesion (hazard ratio, $0.63 ; 95 \%$ confidence interval, 0.59-0.68; $\mathrm{P}<0.001)$, including the bone, brain, liver and lung metastatic sites. However, for patients with two metastatic sites, RT did not improve CSS regardless of metastasis pattern
\end{abstract}

Correspondence to: Dr Haiyong Wang, Department of Internal Medicine-Oncology, Shandong Cancer Hospital and Institute, Shandong First Medical University and Shandong Academy of Medical Sciences, 440 Jiyan Road, Jinan, Shandong 250117, P.R. China

E-mail: wanghaiyong6688@126.com

*Contributed equally

Abbreviations: ES-SCLC, extensive stage small cell lung cancer; SEER, Surveillance Epidemiology and End Results; SCLC, small cell lung cancer; ES, extensive stage; CSS, cancer-specific survival; RT, radiotherapy; TRT, thoracic RT; OS, overall survival; AJCC, American Joint Committee of Cancer; HR, hazard ratio; CI, confidence interval; PCI, prophylactic cranial irradiation; BM, bone metastasis

Key words: small cell lung cancer, extensive-stage, radiotherapy, metastasis pattern, cancer-specific survival (all $\mathrm{P}>0.05$ ). To conclude, RT may improve the survival rate of patients with ES-SCLC with distant metastasis, particularly in those with only one metastatic site.

\section{Introduction}

Small cell lung cancer (SCLC) accounts for 13-20\% of all types of lung cancer $(1,2)$. Due to its short tumor doubling time and invasiveness, the majority of patients present with extensive-stage (ES) disease at the time of diagnosis. The prognosis of patients with ES-SCLC is poor, with a 2-year survival rate $<5 \%$ (1). Although $\sim 70 \%$ of patients respond to treatment with etoposide combined with cisplatin, a combination that is considered to be the cornerstone of chemotherapy for ES-SCLC $(3,4)$, the median survival rate remains poor, at only 7-11 months (5). Notably, studies that have focused on molecular-targeted drugs or more intensive chemotherapy strategies did not adequately improve the survival $(6,7)$.

Radiation therapy (RT) plays an important role in the management of SCLC in both the curative and palliative setting (8). Thoracic RT (TRT) may be a treatment option for patients with ES-SCLC. Jeremic et al (9) demonstrated that the addition of TRT to ES-SCLC treatment led to improved survival compared with patients who underwent chemotherapy alone (9). Since then, a number of studies have focused on the role of TRT for patients with ES-SCLC, and suggested that TRT could improve the prognosis of these patients (10-12). Recently, the results of the Chest Radiotherapy Extensive-Stage Small Cell Lung Cancer Trial study demonstrated that TRT could improve progression-free survival and 2-year overall survival (OS) (13). It also indicated that TRT should be considered for all patients with ES-SCLC who respond to chemotherapy. However, to the best of our knowledge, there is currently little relevant research examining the prognosis of patients who receive $\mathrm{RT}$.

In addition to intrathoracic metastasis, the probability of extrathoracic metastasis of SCLC is also very high. Approximately two-thirds of patients with SCLC develop metastatic diseases, most commonly in the liver, brain and bone. These patients exhibit poor prognosis, with an average survival time of $<10$ months (14). As reported, $10 \%$ of patients 
with SCLC possessed brain metastases when initially diagnosed (2) and 17.5-20.3\% of patients with SCLC developed liver metastasis $(15,16)$. Nakazawa et al (17) also demonstrated that among patients with distant metastasis, 20.3, 18.3, 15.5 and $10.0 \%$ had liver, bone, brain and lung metastasis, respectively. The current treatment method for ES-SCLC with distant metastasis is primarily chemotherapy (18). Worse still, further treatment had a limited effect with a very low response rate to the second-line chemotherapy; therefore, patients usually succumbed to uncontrolled tumor growth $(19,20)$. Previous studies have demonstrated that whole liver RT, external beam RT and whole brain RT in patients with distant metastasis played roles in relieving symptoms. Shamp et al (21) revealed that any patients with external beam radiation could improve OS in those with ES-SCLC. RT may become an effective treatment for patients with ES-SCLC with distant metastasis. However, no clinical studies are currently available with respect to the effect of RT for these patients.

The present study assessed whether RT could improve the survival of patients with ES-SCLC with distant metastasis. RT was defined as RT to a local site regardless of location treatment of ES-SCLC with distant metastasis.

\section{Patients and methods}

Data source. The SEER Cancer Statistics Review (http://seer. cancer.gov/data/citation.html) is published annually by the Data Analysis and Interpretation Branch of the National Cancer Institute (22). The SEER*Stat software (SEER*Stat 8.3.4) was used to identify appropriate patients to be included in the present study. Using this software, and according to the American Joint Committee on Cancer (AJCC) seventh edition Tumor-Node-Metastasis (TNM) stage (23), the present study screened patients that had been diagnosed with ES-SCLC between 2010 and 2013. The inclusion criteria for the included patients were: i) Their ES-SCLC diagnosis was confirmed microscopically; ii) the reported age was accurate; iii) patients attended follow-up appointments; iv) had only one primary tumor; v) were AJCC stage M1 and vi) the exact location of metastasis was known. Due to the SEER database limitations, patient data on when metastasis was diagnosed and whether patients received other treatments for the local disease or metastasis, such as chemotherapy, was unknown. Other information regarding the functional ability of patients [e.g., Eastern Cooperative Oncology Group (ECOG) scores] or comorbidities was not recorded. Patients with benign or borderline tumors, those lacking information on age, metastasis information, RT information, cause of death and survival time were excluded. In addition, if it was not clear whether metastasis occurred in the lung, bone, liver or brain, or if metastasis occurred in a different site, the patients were also excluded.

Ethics statement. The present study was performed in compliance with the Declaration of Helsinki. Permission was obtained to access the SEER program research data. Informed consent was not required as personal identifying information was not included. The present study was approved by the Ethics Committee of the Shandong Cancer Hospital and Institute, Shandong First Medical University and Shandong Academy of Medical Sciences. (Jinan, China).
Statistical analysis. The following variables were analyzed for all patients: Age, ethnicity, sex, AJCC T, AJCC N, bone metastasis, brain metastasis, lung metastasis and liver metastasis. Cancer-specific survival (CSS) was regarded as the primary endpoint of the present study. 'Prognosis factor' refers to all factors affecting the outcome of SCLC in the present study. The $\chi^{2}$ test was used to compare the patient baseline characteristics. Kaplan-Meier analysis was used to generate the survival curves and the log-rank test was applied to analyze the differences. Finally, univariate and multivariate Cox proportional hazard regression models were used to evaluate the association between different variables and CSS in patients with ES-SCLC exhibiting distant metastasis. Based on the multivariate analysis, a forest plot was created to analyze the subgroups. In the present study, 'metastasis number' was defined as the number of metastatic sites involved regardless of the number of metastatic lesions within each organ. All statistical tests were two-sided, and $\mathrm{P}<0.05$ was considered to indicate a statistically significant difference. SPSS software (version 18.0; SPSS, Inc.) and the R statistical package (version 3.1.1; Project for Statistical Computing) were used for the data analysis.

\section{Results}

Patient characteristics. A total of 8,595 patients with ES-SCLC who met the inclusion criteria and were treated between 2010 and 2013, were taken from the SEER database for analysis. In total, $39.5 \%$ of patients received RT. In addition, the majority of patients were diagnosed with SCLC at $>49$ years of age $(95.8 \%)$ and were white $(87.2 \%)$. As presented in Table I, 35.6\% of the patients had stage T4 disease and $54.9 \%$ had stage N2, according to the AJCC staging system. A significant difference was observed in variables including age, ethnicity, AJCC T stage, AJCC N stage, brain metastasis, lung metastasis and liver metastasis (all $\mathrm{P}<0.05$ ). However, this difference was not observed among certain other variables, including sex $(\mathrm{P}=0.710)$ and bone metastasis $(\mathrm{P}=0.258)$. Compared with the RT group, there was a lower number of patients that had brain metastasis who did not receive RT (13.5\% vs. $54.2 \%$; $\mathrm{P}<0.001)$, and a greater number of patients that had liver metastasis $(68.1 \%$ vs. $39.6 \%$; $\mathrm{P}<0.001)$ and lung metastasis $(27.0 \%$ vs. $21.7 \%$; $\mathrm{P}<0.001)$.

Survival outcome analysis. Kaplan-Meier analysis was used to analyze patient survival rate. The results revealed that patients treated with RT had better outcomes with significantly improved overall survival and CSS rates $(\mathrm{P}<0.001)$ compared with those who did not receive RT (Fig. 1A-B). Univariate and multivariate Cox proportional hazard regression models were used to assess the prognostic value of baseline characteristics in the present study. The results, presented in Table II, revealed that RT was an independent prognostic factor affecting patient CSS [hazard ratio (HR), 1.591; 95\% confidence interval (CI), 1.513-1.672; $\mathrm{P}<0.001)$. In addition, other variables including age, ethnicity, sex, AJCC T stage, AJCC N stage and metastatic number also affected the CSS of patients with ES-SCLC (all P<0.05).

Subgroup analysis regarding the effect of the number of metastatic sites on CSS. The subgroup from the multivariate 
Table I. Patient characteristics of ES-SCLC with distant metastasis from the SEER Database.

\begin{tabular}{|c|c|c|c|c|}
\hline Characteristics & Total $(\%)$ & Radiotherapy (\%) & No radiotherapy (\%) & P-value \\
\hline Total & $8,595(100)$ & $3,397(39.5)$ & $5,198(60.5)$ & \\
\hline Age, years & & & & $<0.001$ \\
\hline$\leq 49$ & $360(4.2)$ & $202(5.9)$ & $158(3.0)$ & \\
\hline $50-64$ & $3,248(37.8)$ & $1,486(43.7)$ & $1,762(33.9)$ & \\
\hline$\geq 65$ & 4,987 (58.0) & $1,709(50.3)$ & $3,278(63.1)$ & \\
\hline Ethnicity & & & & 0.004 \\
\hline White & $7,495(87.2)$ & $2,912(85.7)$ & $4,583(88.2)$ & \\
\hline Black & $767(8.9)$ & 337 (9.9) & $430(8.3)$ & \\
\hline Other & $333(3.9)$ & $148(4.4)$ & $185(3.6)$ & \\
\hline Sex & & & & 0.710 \\
\hline Male & $4,614(53.7)$ & $1,832(53.9)$ & $2,782(53.5)$ & \\
\hline Female & $3,981(46.3)$ & $1,565(46.1)$ & $2,416(46.5)$ & \\
\hline AJCC T stage & & & & $<0.001$ \\
\hline $\mathrm{T} 1$ & $755(8.8)$ & $331(9.7)$ & $424(8.2)$ & \\
\hline $\mathrm{T} 2$ & $1,809(21.0)$ & $699(20.6)$ & $1,110(21.4)$ & \\
\hline $\mathrm{T} 3$ & $1,644(19.1)$ & $666(19.6)$ & $978(18.8)$ & \\
\hline $\mathrm{T} 4$ & $3,062(35.6)$ & $1,314(38.7)$ & $1,748(33.6)$ & \\
\hline $\mathrm{Tx}$ & $1,325(15.4)$ & $387(11.4)$ & $938(18.0)$ & \\
\hline AJCC N stage & & & & 0.002 \\
\hline No & $1,006(11.7)$ & $397(11.7)$ & 609 (11.7) & \\
\hline N1 & $576(6.7)$ & $247(7.3)$ & $329(6.3)$ & \\
\hline $\mathrm{N} 2$ & $4,720(54.9)$ & $1,840(54.2)$ & $2,880(55.4)$ & \\
\hline N3 & $1,863(21.7)$ & $775(22.8)$ & $1,088(20.9)$ & \\
\hline $\mathrm{Nx}$ & $430(5.0)$ & $138(4.1)$ & $292(5.6)$ & \\
\hline Bone metastasis & & & & 0.258 \\
\hline Yes & $3,577(41.6)$ & $1,439(42.4)$ & $2,138(41.1)$ & \\
\hline No & $5,018(58.4)$ & $1,958(57.6)$ & $3,060(58.9)$ & \\
\hline Brain metastasis & & & & $<0.001$ \\
\hline Yes & $2,542(29.6)$ & $1,842(54.2)$ & $700(13.5)$ & \\
\hline No & $6,053(70.4)$ & $1,555(45.8)$ & 4,498 (86.5) & \\
\hline Lung metastasis & & & & $<0.001$ \\
\hline Yes & $2,137(24.9)$ & $736(21.7)$ & $1,401(27.0)$ & \\
\hline No & $6,458(75.1)$ & $2,661(78.3)$ & $3,797(73.0)$ & \\
\hline Liver metastasis & & & & $<0.001$ \\
\hline Yes & $4,884(56.8)$ & $1,346(39.6)$ & $3538(68.1)$ & \\
\hline No & $3,711(43.2)$ & $2,051(60.4)$ & $1,660(31.9)$ & \\
\hline
\end{tabular}

ES-SCLC, extensive stage small cell lung cancer; SEER, Surveillance Epidemiology and End Results; AJCC, American Joint Committee on Cancer; T, Tumor; N, Node.

analysis was then used for the forest plot, and variables including age, ethnicity, sex, AJCC stage T, AJCC stage N and metastasis number were analyzed (Fig. 2). The forest plots revealed that all subgroups favored RT. A metastasis number of one was a significant prognosis factor for patients with ES-SCLC receiving RT (HR, 0.63; 95\% CI, 0.59-0.68; $\mathrm{P}<0.001)$. It also revealed that a metastasis number $>1$ had an impact on RT (HR, 0.90; 95\% CI, 0.82-0.98; $\mathrm{P}=0.021$ ). According to the P-values, patients with one metastatic site benefited more from radiotherapy compared with those with multiple metastatic sites. Overall, the results of the forest plot demonstrated that all subgroups, except age $\leq 49$ subgroup (HR, 0.80; 95\% CI, 0.61-1.06; $\mathrm{P}=0.113$ ) were in favor of RT.

Survival outcome analysis of patients with only one metastasis site. Kaplan-Meier analysis was used to evaluate the survival of patients with only bone, brain, liver or lung metastasis. The results revealed that RT could improve CSS in all patients with only one metastasis site regardless of metastatic 
Table II. Influence of different variables on cancer specific survival for patients with ES-SCLC analyzed by Cox proportional hazard model.

\begin{tabular}{|c|c|c|c|c|c|}
\hline \multirow[b]{2}{*}{ Variable } & \multicolumn{2}{|c|}{ Univariate analysis } & \multicolumn{3}{|c|}{ Multivariate analysis } \\
\hline & $\chi^{2}$ & P-value & HR & $95 \% \mathrm{CI}$ & P-value \\
\hline Age, years & 152.30 & $<0.001$ & & & $<0.001$ \\
\hline$\leq 49$ & & & Reference & & \\
\hline $50-64$ & & & 1.093 & $0.966-1.236$ & 0.158 \\
\hline$\geq 65$ & & & 1.378 & $1.220-1.555$ & $<0.001$ \\
\hline Ethnicity & 12.70 & 0.002 & & & 0.006 \\
\hline White & & & Reference & & \\
\hline Black & & & 0.954 & $0.877-1.038$ & 0.274 \\
\hline Others & & & 0.814 & $0.714-0.928$ & 0.002 \\
\hline Sex & 27.70 & $<0.001$ & & & $<0.001$ \\
\hline Male & & & Reference & & \\
\hline Female & & & 0.890 & $0.849-0.934$ & $<0.001$ \\
\hline AJCC T stage & 27.96 & $<0.001$ & & & 0.008 \\
\hline $\mathrm{T} 1$ & & & Reference & & \\
\hline $\mathrm{T} 2$ & & & 1.142 & $1.037-1.258$ & 0.007 \\
\hline $\mathrm{T} 3$ & & & 1.147 & $1.040-1.266$ & 0.006 \\
\hline $\mathrm{T} 4$ & & & 1.150 & $1.050-1.260$ & 0.003 \\
\hline AJCC N stage & 27.12 & $<0.001$ & & & 0.003 \\
\hline N0 & & & Reference & & \\
\hline N1 & & & 0.953 & $0.848-1.071$ & 0.417 \\
\hline $\mathrm{N} 2$ & & & 1.059 & $0.981-1.144$ & 0.143 \\
\hline N3 & & & 0.973 & $0.891-1.062$ & 0.533 \\
\hline Metastatic lesions & 78.73 & $<0.001$ & & & $<0.001$ \\
\hline 1 & & & Reference & & \\
\hline$>1$ & & & 1.237 & $1.178-1.299$ & $<0.001$ \\
\hline Radiotherapy & 452.61 & $<0.001$ & & & $<0.001$ \\
\hline Yes & & & Reference & & \\
\hline No & & & 1.591 & $1.513-1.672$ & $<0.001$ \\
\hline
\end{tabular}

ES-SCLC, extensive stage small cell lung cancer; HR, hazard ratio, CI, confidence interval; AJCC, American Joint Committee on Cancer; T, Tumor; N, Node.
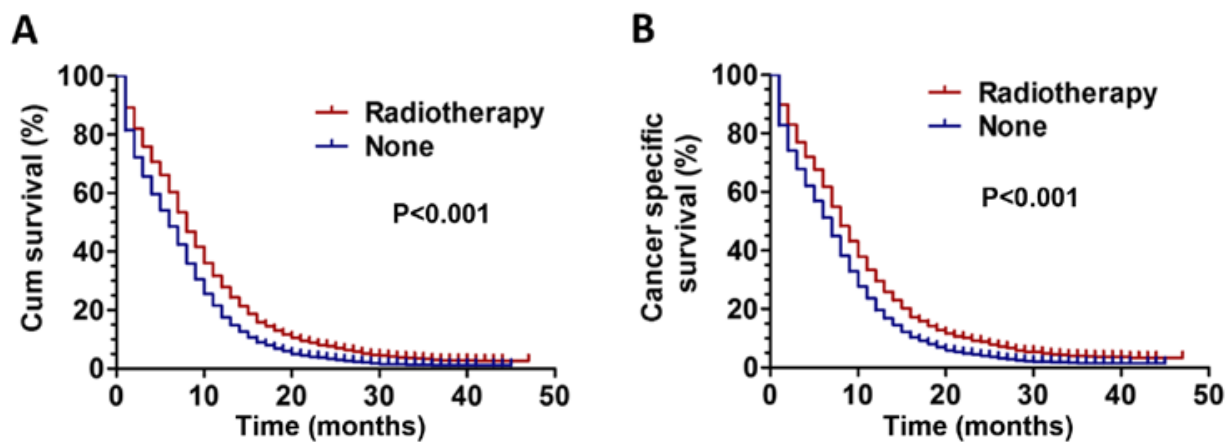

Figure 1. (A) Survival curves for overall survival in patients with ES-SCLC between RT and non-RT groups. (B) Survival curves for CSS in patients with ES-SCLC between RT and non-RT groups. Cum survival, cumulative overall survival; CSS, cancer-specific survival; ES-SCLC, extensive stage small cell lung cancer; RT, radiotherapy.

pattern (bone metastasis, brain, liver and lung metastasis, all $\mathrm{P}<0.001$ (Fig. 3A-D).
Survival outcomes analysis of patients with more than one metastasis site. Further analysis of patients with more than 


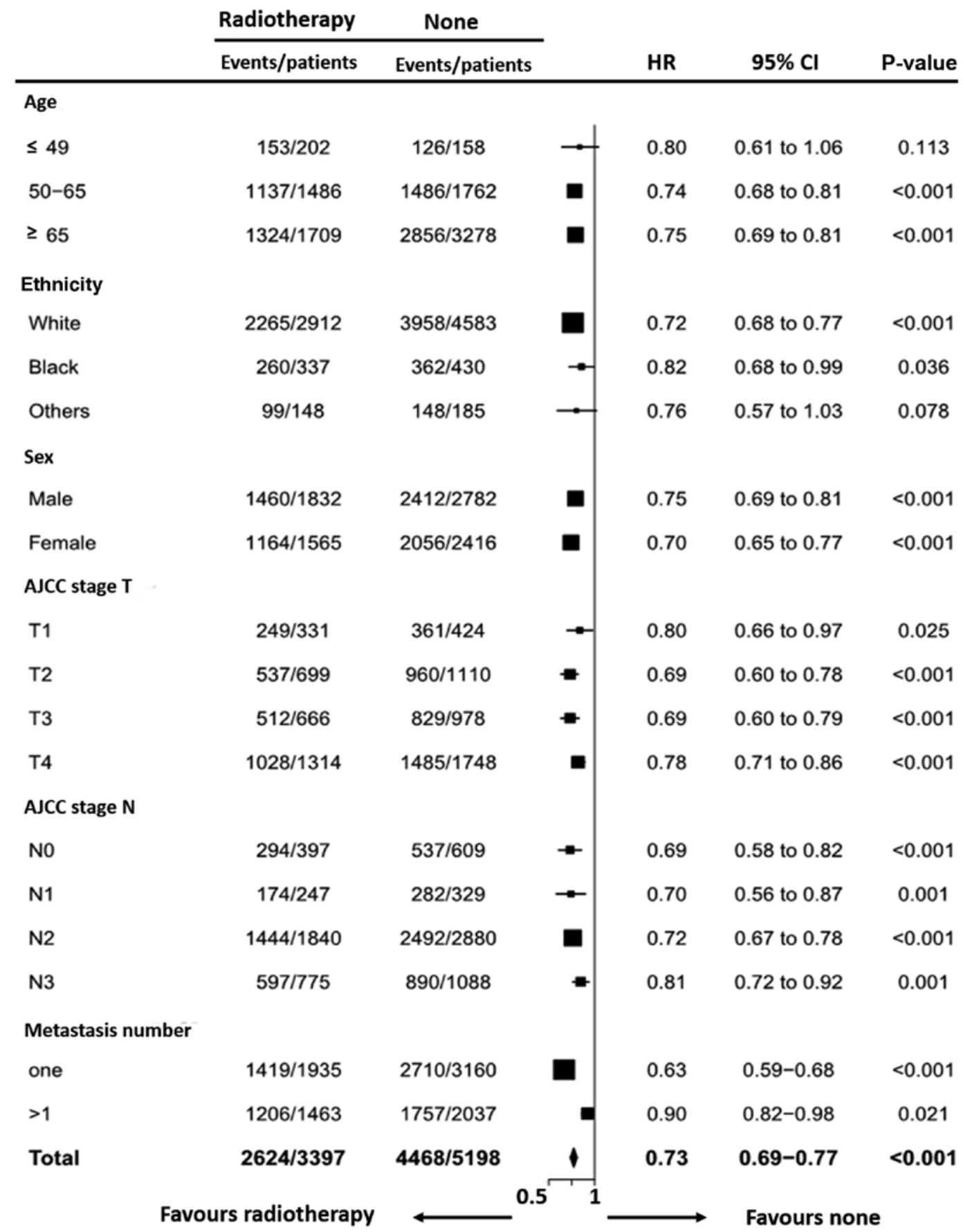

Figure 2. Forest plot based on the multivariate analysis for the HRs between the RT group and non-RT group according to different variables. HR, hazard ratio; RT, radiotherapy; CI, confidence interval.

one metastatic site was performed. Kaplan-Meier analysis was used to analyze survival. For patients with two metastatic sites, RT did not improve CSS: Bone + brain metastasis, $\mathrm{P}=0.647$ (Fig. 4A); bone + liver metastasis, $\mathrm{P}=0.227$ (Fig. 4B); brain + liver metastasis, $\mathrm{P}=0.242$ (Fig. $4 \mathrm{C}$ ); bone + lung metastasis, $\mathrm{P}=0.334$ (Fig. 4D); brain + lung metastasis, $\mathrm{P}=0.075$ (Fig. 4E); liver + lung metastasis, $\mathrm{P}=0.239$ (Fig. 4F). Additionally, patients with three metastatic sites were analyzed, and it was revealed that RT did not improve CSS: Bone + brain + liver metastasis, $\mathrm{P}=0.095$ (Fig. S1A); brain + liver + lung metastasis, $\mathrm{P}=0.217$ (Fig. S1D); but not in patients with bone + brain + lung metastasis, $\mathrm{P}=0.001$ (Fig. S1B) and bone + liver + lung metastasis, $\mathrm{P}=0.042$ (Fig. $1 \mathrm{SC}$ ).

\section{Discussion}

RT has been demonstrated as an effective treatment method for SCLC $(24,25)$. An increasing amount of evidence has been presented that supports the application of TRT in ES-SCLC. The 5-year OS rate for complete responders has been improved from 3.7 to $9.1 \%$ by TRT, and the local recurrence rate was also significantly decreased, as reported by Jeremic et al in (9). Slotman et al (13) investigated the role of TRT in addition to PCI for patients with ES-SCLC who responded to chemotherapy. Although failure was observed in survival at 1 year (33\% vs. 28\%), 2-year overall survival (13\% vs. 3\%) was significantly improved. However, the extent to which the 

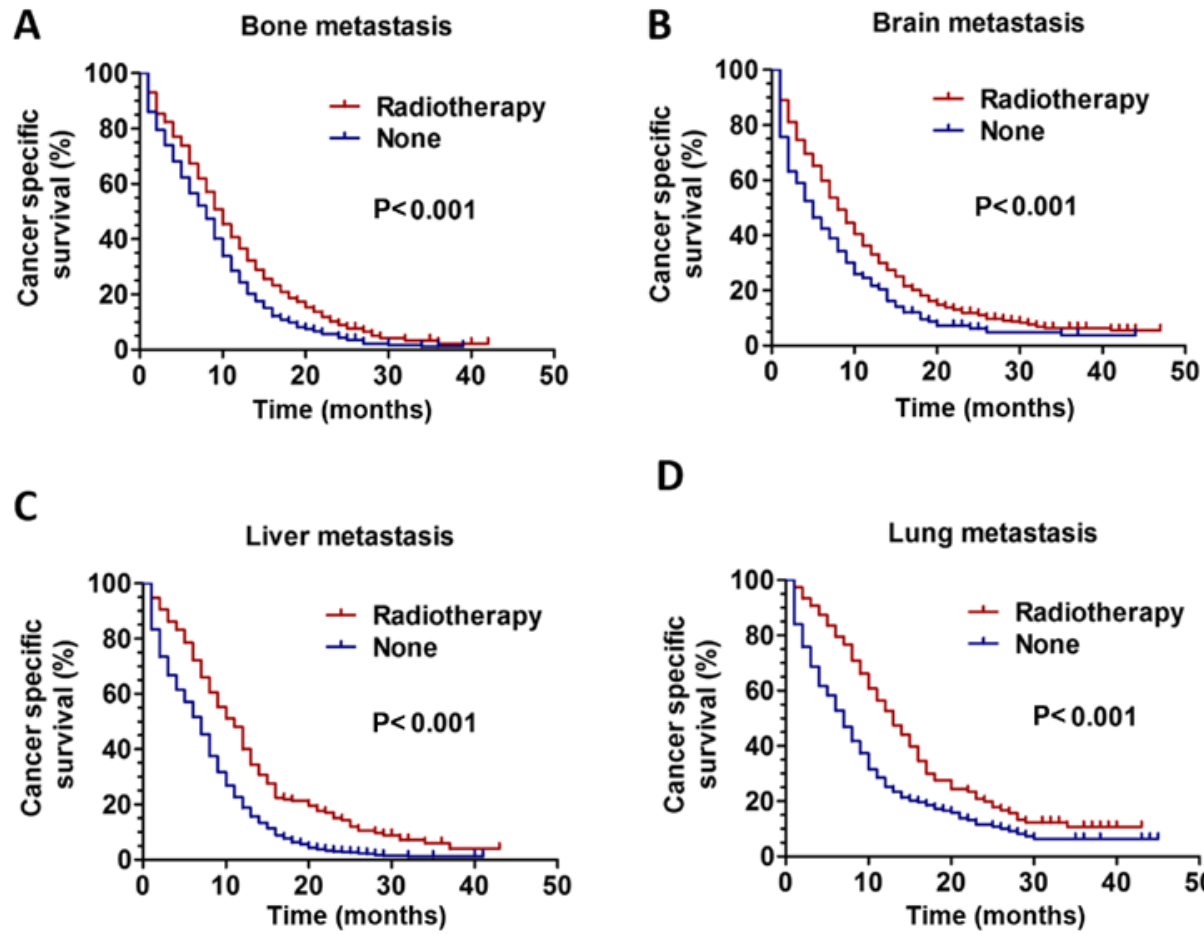

D

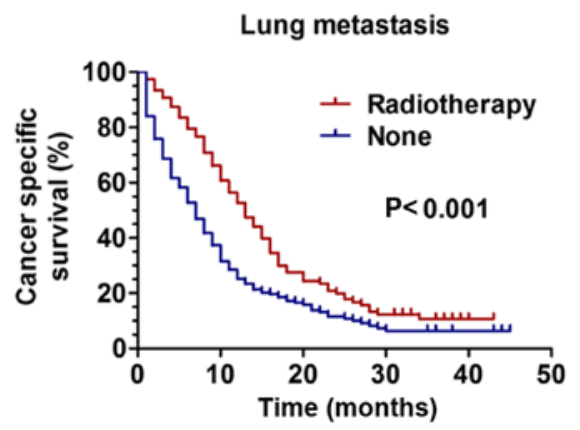

Figure 3. CSS curves for patients with ES-SCLC between the RT and non-RT groups based on only one metastasis site. The CSS curves for patients with (A) only bone metastasis $(\mathrm{P}<0.001)$, (B) only brain metastasis $(\mathrm{P}<0.001)$, (C) only liver metastasis $(\mathrm{P}<0.001)$ and $(\mathrm{D})$ only lung metastasis $(\mathrm{P}<0.001)$. CSS cancer-specific survival; ES-SCLC, extensive stage small cell lung cancer; RT, radiotherapy.

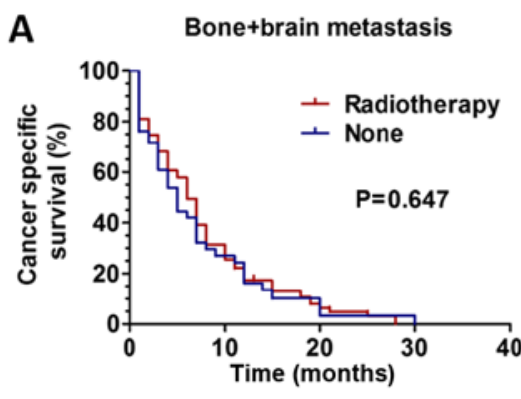

D

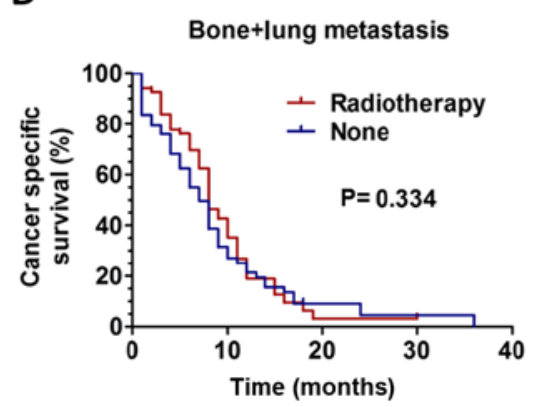

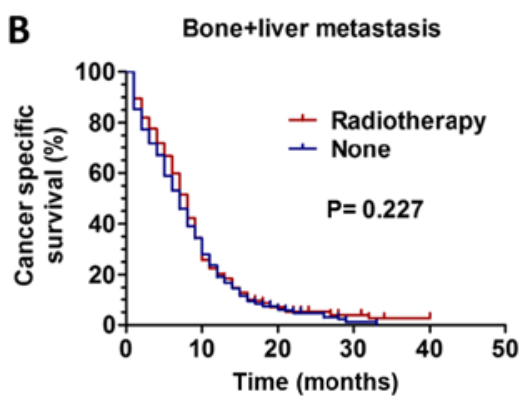

E

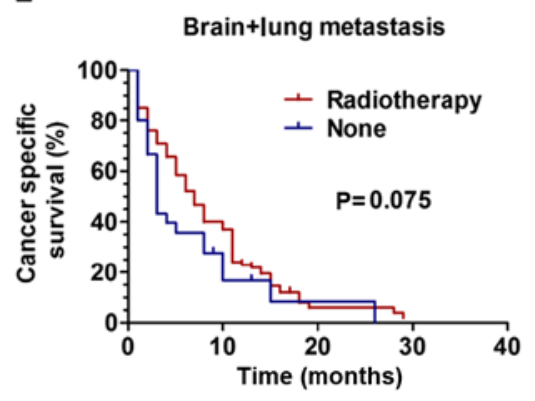

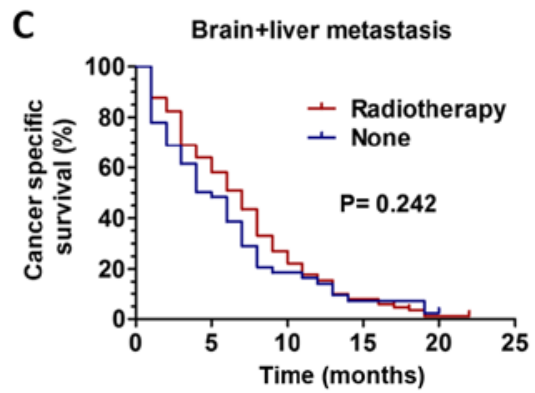

F

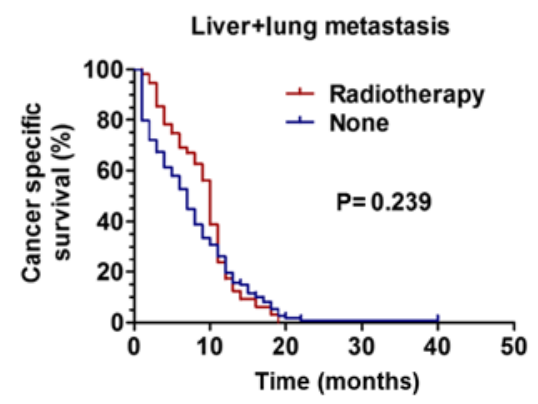

Figure 4. CSS curves for patients with ES-SCLC between the RT and non-RT groups based on two metastasis sites. The CSS curves for patients with (A) bone + brain metastasis $(\mathrm{P}=0.647),(\mathrm{B})$ bone + liver metastasis $(\mathrm{P}=0.227),(\mathrm{C})$ brain + liver metastasis $(\mathrm{P}=0.242),(\mathrm{D})$ bone + lung metastasis $(\mathrm{P}=0.334),(\mathrm{E})$ brain + lung metastasis $(\mathrm{P}=0.075)$ and $(\mathrm{F})$ liver + lung metastasis $(\mathrm{P}=0.239)$. CSS, cancer-specific survival; ES-SCLC, extensive stage small cell lung cancer; RT, radiotherapy.

TRT survival improvement was different from that reported by Slotman et al (13) may be attributable to population diversity. This population was not exclusive of the patients with brain metastasis, but also in all those who received PCI. In the present study, $29.6 \%$ of patients presented with brain metastasis, which was a significant negative predictor of survival.
In SCLC, $17.5-20.3 \%$ of patients developed liver metastasis, with a median survival time of 3 months (26). Tas et al (27) reported that the incidence of liver metastasis in patients with lung cancer was $37-51 \%$ on autopsy. Current use in symptomatic patients of whole liver RT may offer only symptomatic relief, and has long been abandoned as an anti-cancer therapy (28). 
Soliman et al (29) demonstrated that single fraction of external beam RT directed to the whole liver for patients experiencing painful liver metastasis could achieve meaningful symptom relief and improved quality of life in the majority of patients. Consistently, in the present study, patients with RT had better outcomes with significantly improved CSS compared with the patients receiving non-RT. Furthermore, the impact that the RT side effects have on the quality of life of these patients should also be considered. In addition, lung cancer has been demonstrated to cause bone metastasis (BM) in $40-80 \%$ of patients (30). At present, the primary goals for the treatment of $\mathrm{BM}$ are pain relief, preservation of mobility and function, prevention of future complications, and optimized quality of life. Notably, RT could improve CSS in patients with ES-SCLC with only BM. This result may guide clinicians to treat the patients correctly, and may exhibit important clinical significance.

Remarkably, $\sim 60 \%$ of patients with SCLC develop brain metastasis. The prognosis of brain metastasis is particularly poor, with a median survival time of $<1$ year. The local control rate of brain metastasis by whole brain RT alone is $\sim 50 \%$, and the 1-year survival rate is only $10-20 \%(31,32)$. Similarly, in the present study, the results of the data analysis also demonstrated that RT could improve CSS in patients with ES-SCLC with only brain metastasis.

Until recently, there was little relevant research regarding the impact of RT on the survival of patients with ES-SCLC that exhibited distant metastasis. The present study investigated the prognosis of patients who received RT based on their distant metastasis pattern. The results indicated that patients with only one distant metastatic site, including bone, brain, liver and lung, could improve the CSS with administration of RT, while the patients with ES-SCLC with more than one metastasis site may not benefit from RT regardless of the metastasis pattern, except those patients with bone + brain + lung metastasis, bone + liver + lung metastasis, which may be due to the minimal sample size. The survival curves of patients with bone + liver + lung metastasis demonstrated a slight difference. Although this result was statistically significant, it had no clinical significance due to the low sample size. The forest plot revealed that the number of metastatic lesions was a significant prognostic factor for patients with ES-SCLC receiving $\mathrm{RT}$ with one or multiple metastatic sites.

The reasons that more than one metastasis number exhibited statistical significance may be as follows: One plausible explanation was that in patients with three metastatic sites, despite the statistical significance, the overall statistical value was affected by the minimal sample size. The results were statistically significant, but there was no clinical significance. Another possible explanation may be that, for the patients with more than one distant metastasis, RT only played a role in relieving symptoms, but could not improve prognosis. In addition, intrathoracic tumor progression may play a major role in the mortality of patients with ES-SCLC with only one metastasis. When two or more organs are involved, progression of extrathoracic sites may lead to greater damage to the body than the intra-thoracic site, and the overall mortality is not decreased by the RT.

This present study had several limitations that should be noted. First, the results may be weakened due to information that was not available, including details on when the RT was delivered and other systemic treatments given to the patients. It was not possible to obtain the information regarding treatment history with cytotoxic drugs, the response to treatment and general conditions, such as performance status, which are essential for the analysis of patient survival. As combined PCI or TRT are not recorded in the SEER database, the impact of aggressive multimodality approach to treatment on survival could also not be analyzed. In addition, the volume of the metastasis site, which reflects disease burden, is not recorded in the database and so no analysis using this variable was possible. Similarly, patient information regarding intrathoracic RT was unavailable. Finally, due to the constraints of the SEER database, it was not possible to obtain certain specific information, including other treatments, functional ability of patients or ECOG scores. In addition, it would have been ideal to measure overall survival; however, due to the limitations of the database, it was not possible to obtain the exact survival time. These variables should be investigated in future studies. The limitation of not having this information in the analysis of patient survival in detail had some impacts on the conclusion of the present study, and therefore, the conclusions drawn may be not reliable without this information. Another limitation may be that the median survival time for patients with and without RT for each of the metastatic patterns was not calculated in the present study. Further prospective studies to determine whether RT can improve the prognosis for the patients with ES-SCLC with only one metastasis site are necessary.

The results of the present study indicated that RT significantly improved CSS in patients with only one metastasis site, including bone, brain, liver and lung. However, for patients with two or more metastasis sites, RT did not improve CSS regardless of metastasis pattern.

\section{Acknowledgements}

Not applicable.

\section{Funding}

No funding was received.

\section{Availability of data and materials}

The datasets used and/or analyzed during the present study are available from the corresponding author on reasonable request.

\section{Authors' contributions}

XS analyzed the data and wrote the manuscript. JL and ZL acquired and analyzed the data. HW designed the study and participated in data analysis and interpretation.

\section{Ethics approval and consent to participate}

The present study was approved by the Ethics Committee of the Shandong Cancer Hospital and Institute, Shandong First Medical University and Shandong Academy of Medical Sciences. 


\section{Patient consent for publication}

Not applicable.

\section{Competing interests}

The authors declare that they have no competing interests.

\section{References}

1. Govindan R, Page N, Morgensztern D, Read W, Tierney R, Vlahiotis A, Spitznagel EL and Piccirillo J: Changing epidemiology of small-cell lung cancer in the United States over the last 30 days: Analysis of the surveillance, epidemiologic, and end results database. J Clin Oncol 24: 4539-4544, 2006.

2. van Meerbeeck JP, Fennell DA and De Ruysscher DK: Small-cell lung cancer. Lancet 378: 1741-1755, 2011.

3. Jett JR, Schild SE, Kesler KA and Kalemkerian GP: Treatment of small cell lung cancer: Diagnosis and management of lung cancer, 3rd edn: American College of Chest Physicians evidence-based clinical practice guidelines. Chest 143 (Suppl 5): e400S-e419S 2013.

4. Früh M, De Ruysscher D, Popat S, Crinò L, Peters S and Felip E; ESMO Guidelines Working Group: Small-cell lung cancer (SCLC): ESMO Clinical Practice Guidelines for diagnosis, treatment and follow-up. Ann Oncol 24 (Suppl 6): vi99-vi105, 2013

5. Eckert F and Muller AC: SCLC-extensive disease-treatment guidance by extent or/and biology of response? Radiat Oncol 3: 33, 2008.

6. Kalemkerian GP: Advances in pharmacotherapy of small cell lung cancer. Expert Opin Pharmacother 15: 2385-2396, 2014

7. Riess JW and Lara PN Jr: Left behind? Drug discovery in extensive-stage small-cell lung cancer. Clin Lung Cancer 15 93-95, 2014.

8. Glatzer M, Schmid S, Radovic M, Früh M and Putora PM: The role of radiation therapy in the management of small cell lung cancer. Breathe (Sheff) 13: e87-e94, 2017.

9. Jeremic B, Shibamoto Y, Nikolic N, Milicic B, Milisavljevic S, Dagovic A, Aleksandrovic J and Radosavljevic-Asic G: Role of radiation therapy in the combined-modality treatment of patients with extensive disease small-cell lung cancer. a randomized study. J Clin Oncol 17: 2092-2099, 1999.

10. Giuliani ME, Atallah S, Sun A, Bezjak A, Le LW, Brade A, Cho J, Leighl NB, Shepherd FA and Hope AJ: Clinical outcomes of extensive stage small cell lung carcinoma patients treated with consolidative thoracic RT. Clin Lung Cancer 12: 375-379, 2011.

11. Zhu H, Zhou Z, Wang Y, Bi N, Feng Q, Li J, Lv J, Chen D, Shi Y and Wang L: Thoracic radiation therapy improves the overall survival of patients with extensive-stage small cell lung cancer with distant metastasis. Cancer 117: 5423-5431, 2011.

12. Yee D, Butts C, Joy A, Smylie M, Fenton D, Chu Q, Hanson J and Roa W: Clinical trial of post-chenotherapy consolidation thoracic radiotherapy for extensive-stage small cell lung cancer. Radiother Oncol 102: 234-238, 2012.

13. Slotman BJ, van Tinteren H, Praag JO, Knegjens JL, El Sharouni SY, Hatton M, Keijser A, Faivre-Finn C and Senan S: Use of thoracic RT for extensive stage small-cell lung cancer: A phase 3 randomised controlled trial. Lancet 385: 36-42, 2015.

14. Masters GA: Clinical presentation of small-cell lung cancer. In: Pass HI, Carbone DP, Johnson DH, Minna JD, Scagliotti GV Turrissi AT (eds) Principles and practice of lung cancer: The official reference text of the international association for the study of lung cancer (IASLC), 4th edn. Lippincot Williams and Wilkins, Philadelphia, pp 341-352, 2010.

15. Stenbygaard LE, Sørensen JB, Larsen H and Dombernowsky P: Metastatic pattern in non-resectable non-small cell lung cancer. Acta Oncol 38: 993-998, 1999.

16. Kagohashi K, Satoh H, Ishikawa H, Ohtsuka M and Sekizawa K: Liver metastasis at the time of initial diagnosis of lung cancer. Med Oncol 20: 25-28, 2003.
17. Nakazawa K, Kurishima K, Tamura T, Kagohashi K, Ishikawa H, Satoh $\mathrm{H}$ and Hizawa N: Specific organ metastases and survival in small cell lung cancer. Oncol Lett 4: 617-620, 2012.

18. Horn L, Castellanos EL and Johnson DH: Update on new drugs in small cell lung cancer. Expert Opin Investig Drugs 20: 441-445, 2011.

19. Lara PN Jr, Natale R, Crowley J, Lenz HJ, Redman MW, Carleton JE, Jett J, Langer CJ, Kuebler JP, Dakhil SR, et al: Phase III trial of irinotecan/cisplatin compared with etoposide/cisplatin in extensive-stage small-cell lung cancer: Clinical and pharmacogenomic results from SWOG S0124. J Clin Oncol 27: 2530-2535, 2009.

20. Koinis F, Agelaki S, Karavassilis V, Kentepozidis N, Samantas E, Peroukidis S, Katsaounis P, Hartabilas E, Varthalitis II, Messaritakis I, et al: Second-line pazopanib in patients with relapsed and refractory small-cell lung cancer: A multicentre phase II study of the Hellenic oncology research group. $\mathrm{Br}$ J Cancer 117: 8-14, 2017.

21. Shamp S, Patel RB and Biswas T: Palliative radiation therapy in extensive stage small cell lung cancer (ES-SCLC): A survival, epidemiology and end results (SEER) analysis. Int J Radit Oncol Biol 99: e495, 2017.

22. Surveillance, Epidemiology, and End Results (SEER) Program (www.seer.cancer.gov) Research Data (1975-2016), National Cancer Institute, DCCPS, Surveillance Research Program, released April 2019, based on the November 2018 submission.

23. Goldstraw P, Crowley J, Chansky K, Giroux DJ, Groome PA, Rami-Porta R, Postmus PE, Rusch V and Sobin L; International Association for the Study of Lung Cancer International Staging Committee; Participating Institutions: The IASLC Lung Cancer Staging Project: Proposals for the revision of the TNM stage groupings in the forthcoming (seventh) edition of the TNM Classification of malignant tumours. J Thorac Oncol 2: 706-714, 2007.

24. O'Brien ME, Ciuleanu TE, Tsekov H, Shparyk Y, Cuceviá B, Juhasz G, Thatcher N, Ross GA, Dane GC and Crofts T: Phase III trial comparing supportive care alone with supportive care with oral topotecan in patients with relapsed small-cell lung cancer. J Clin Oncol 24: 5441-5447, 2006.

25. Froeschl S, Nicholas G, Gallant V and Laurie SA: Outcomes of second-line chemotherapy in patients with relapsed extensive small cell lung cancer. J Thorac Oncol 3: 163-169, 2008.

26. Wu C, Li F and Jiao SC: Prognostic factors for survival of patients with extensive stage small cell lung cancer-a retrospective single institution analysis. Asian Pac J Cancer Prev 13: 4959-4962, 2012.

27. Tas F, Aydiner A, Topuz E, Camlica H, Saip P and Eralp Y: Factors influencing the distribution of metastases and survival in extensive disease small cell lung cancer. Acta Oncol 38: 1011-1015, 1999.

28. Bydder S, Spry NA, Christie DR, Roos D, Burmeister BH, Krawitz H, Davis S, Joseph DJ, Poulsen M and Berry M: A prospective trial of short-fractionation RT for the palliation of liver metastases. Australas Radiol 47: 284-288, 2003.

29. Soliman H, Ringash J, Jiang H, Singh K, Kim J, Dinniwell R, Brade A, Wong R, Brierley J, Cummings B, et al: Phase II trial of palliative RT for hepatocellular carcinoma and liver metastases. J Clin Oncol Off J Am Soc Clin Oncol 31: 3980-3986, 2013.

30. Morikawa K, Mineshita M, Nishine H, Furuya N, Obayashi J and Miyazawa T: A case of squamous cell lung carcinoma with bone metastasis responding to denosumab after zoledronic acid hydrate. Jap J Lung Cancer 52: 1035-1040, 2012.

31. Khuntia D, Brown P, Li J and Mehta MP: Whole-brain radiotherapy in the management of brain metastasis. J Clin Oncol 24: 1295-1304, 2006

32. Zabel A and Debus J: Treatment of brain metastases from non-small-cell lung cancer (NSCLC): Radiotherapy. Lung Cancer 45 (Suppl 2): S247-S252, 2004.

This work is licensed under a Creative Commons Attribution-NonCommercial-NoDerivatives 4.0 International (CC BY-NC-ND 4.0) License. 